\title{
Approximate Characterization of Large Photovoltaic Power Plants at the Point of Interconnection
}

\author{
A. Bonfiglio, F. Delfino, M. Invernizzi, A. Labella, \\ D. Mestriner and R. Procopio \\ University of Genoa - Dept. of Electrical, Electronic, \\ Telecommunications Engineering and Naval Architecture \\ Via Opera Pia 11a I-16145 Genoa, Italy \\ a.bonfiglio@unige.it
}

\begin{abstract}
The aim of the present article is that of proposing a calculation procedure to assess electric quantities at the Point of Interconnection (POI) of large PhotoVoltaic (PV) power plants on the basis of rated data and main design elements of the plant itself. The quantities of inters are active and reactive power available at the POI in order to extrapolate the power plant capability starting from the capabilities of PWM inverters. The procedure also allows evaluating the POI voltage variations, an important element due to the increasing requirements for renewable generation units to participate in voltage regulation. The main interest in such a methodology lays in its simplicity of application, that allows avoiding the usage of dedicated software for load flow calculations, and flexibility, that makes it suitable for the support of the bidding and pre-design phase of large photovoltaic power plants.
\end{abstract}

Index Terms - Renewable integration, Photovoltaic Power Plants.

\section{INTRODUCTION}

The current trend of evolution of electricity distribution and transmission networks have recorded a strong increasing of distributed generation at all voltage levels. Among these varieties of generation renewables have played a significant role pushed by the increasing sensibility to issues related to sustainable development, environmental awareness and greenhouse gasses reduction policies. As far as renewable generation is concerned the market of PhotoVoltaic (PV) power plants has been one of the most flourishing, together with those of wind and biomass.

In the beginning, PV installations were characterized by small rated power and were connected to LV distribution networks. Then, the variety of installations have also involved larger power plants that needed to be interconnected to the MV distribution infrastructure and, in some cases, to the HV transmission one.

This changing asset of the electricity production mix, more and more characterized by the presence of stochastic power, has led Transmission and Distribution System Operators (TSOs/DSOs) to face new challenges in order to guarantee the reliability and quality of energy supply [1]-[2]. For these

\author{
P. Serra \\ ABB S.p.A. - Power System Division \\ Via Albareto, 35 I-16153 Genoa, Italy
}

reasons, several system operators around the world have introduced specific requirements for the interconnection of renewable generating units at their electricity networks extending the concept of ancillary service to this kind of plants too [3]-[4].

This new requirements of operation for PV power plants allow the electricity system operator to exploit their reactive power capabilities in order to support voltage and increase the quality of electricity supply [5]-[6]. On the other, these requirements introduced significant changes in the way of designing PV power plants especially related to the heterogeneous requests of TSOs and DSO of different states or countries.

The aim of the present article is that of proposing a simple and effective methodology which gives approximated but reliable information about active and reactive power available at the POI in order to have a preliminary check of the compliancy of a proposed PV plant layout in accordance to specific grid code requirements. Since various requirements also ask the plant to participate in voltage regulation it is important to evaluate the impact of the power plant on the POI voltage magnitude. For this reason, the proposed methodology also provides a calculation of voltage variation at the POI, based on the knowledge of the short circuit parameters of the grid.

In the first part of the paper the proposed methodology is going to be fully detailed after a brief description of the typical layout of large PV power plants. Then the procedure results are compared with those of an exact load flow calculation on a test case PV plant layout in various operational assets in order to evaluate the degree of reliability of the proposed methodology.

\section{METHODOLOGY DESCRIPTION}

Before starting with the methodology description, it is important to analyze the general outline of large photovoltaic plants. These plants are typically connected to the HV electricity networks and are usually divided in a well-defined number of identical/similar sub-fields. This choice is generally imposed due to the fact that the plant covers a very large area (the specific surface needed by a PV installation 
can vary between 6 and $7 \mathrm{~m}^{2} / \mathrm{kWp}$ ) that sometimes can exhibit discontinuities in space extension. The sub-field consists of a number of inverters each connected to the LV side of a LV/MV transformer, giving origin to the so-called PV Station (PVS). PVS are connected in an "in and out" configuration along a feeder. If the number of inverters in the sub-field is relevant, it is necessary to build more than one feeder connected to the same sub-field bus in order to contain voltage variations into an admissible range. Sub-fields are then connected to a common bus by means of a dedicated MV cable or line (MV-SI). Between the common bus and the HV POI, a line or cable (MV-CI) and a MV/HV step-up transformer are installed. In some cases, after the step-up transformer, it is possible to have another line or cable (HVCI). The schematic representation of the PV plant is depicted in Fig. 1.

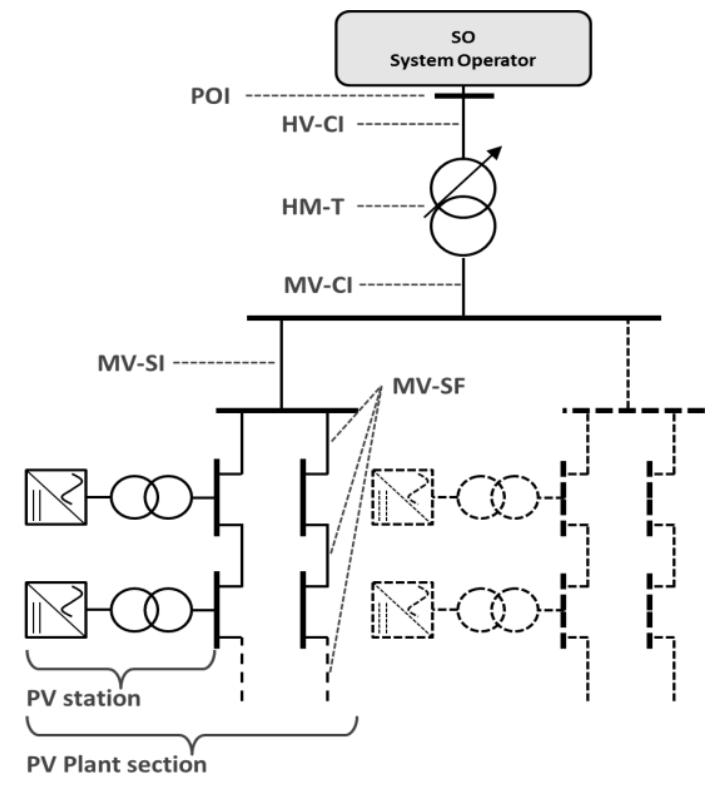

Fig. 1. Large photovoltaic power plants general outline

In the following all equations are written in p.u. considering a power plant common base value for all the subsystems. For the sake of clarity and simplicity, the methodology will be detailed considering the PV plant divided in $N_{s f}$ identical subfields each composed by $N_{f}$ feeders. Feeders are considered to be characterized by the same layout accounting for an identical number of PVS, $N_{i n v}$. It is worth noticing that the proposed methodology can be applied and extended to any other layout of the PV plant giving the possibility to consider asymmetric layouts too.

The procedure starts considering the active and reactive power available at the AC terminal of all the PVSs of the plant. In this contest no specific details are provided for the PWM inverter apart from the check that the given operational point is compatible with the component capability. Given the active and power of the inverter, namely $P_{i n v}$ and $Q_{i n v}$, those quantities have to satisfy the following condition:

$$
\sqrt{P_{i n v}^{2}+Q_{i n v}^{2}} \leq A_{i n v}
$$

If this condition is not satisfied it is necessary to curtail one or both the quantities in order to force operational asset on the capability curve in accordance to specific priorities not considered in the preset formulation.

The subfield collector is the portion which connects each PV inverter to the MV collector bus, as highlighted in Fig. 1. The inputs of this subsystem are all active and reactive powers of the PV plant inverters provided by the inverters. This block accounts for the calculation of the active and reactive power losses in the feeders and subfield interconnection conductors. This section is modeled considering that all voltages are assumed equal to 1 p.u.. On the basis of this assumption, it is possible to mismatch the amplitude of the currents flowing in a generic point to point interconnection with the apparent power flowing in the same branch $A=\sqrt{P^{2}+Q^{2}}$.

Let start considering a generic subfield feeder, consisting of $N_{i n v}$ inverters. First of all, the $k^{\text {th }}$ inverter active and reactive power injections, $P_{i n v, k}$ and $Q_{i n v, k}$, have to be reduced by the active and reactive power losses in the $k^{\text {th }} \mathrm{LV} / \mathrm{MV}$ transformer (respectively $P_{j t k}$ and $Q_{j t k}$ ):

$$
\left\{\begin{array}{l}
P_{j t_{k}}=R_{s c_{k}} \frac{\left(P_{i n v, k}^{2}+Q_{i n v, k}^{2}\right)}{V_{k}} \approx R_{s c_{k}}\left(P_{i n v, k}^{2}+Q_{i n v, k}^{2}\right) k=1 \ldots N_{i n v} \\
Q_{j t_{k}}=X_{s c_{k}} \frac{\left(P_{i n v, k}^{2}+Q_{i n v, k}^{2}\right)}{V_{k}} \approx X_{s c_{k}}\left(P_{i n v, k}^{2}+Q_{i n v, k}^{2}\right) k=1 \ldots N_{i n v}
\end{array}\right.
$$

being $R_{s c k}$ and $X_{s c k}$ respectively the short circuit resistance and reactance of the $k^{\text {th }} \mathrm{LV} / \mathrm{MV}$ transformer. The active and reactive power provided by the generic $k^{\text {th }}$ PVS can then be written as:

$$
\left\{\begin{array}{l}
P_{M V, k}=P_{i n v, k}-P_{j t_{k}} \quad k=1 \ldots N_{i n v} \\
Q_{M V, k}=Q_{i n v, k}-Q_{j t_{k}} \quad k=1 \ldots N_{i n v}
\end{array}\right.
$$

Knowing the active and reactive power available at the MV section of each PVS, it is possible to consider a feeder representation as depicted in Fig. 2:

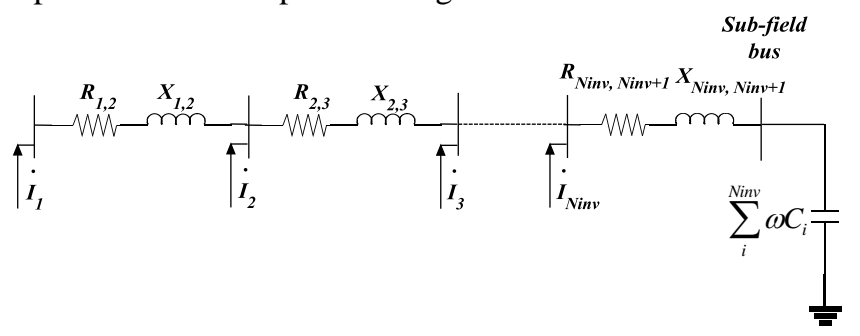

Fig. 2. Feeder circuital representation

Looking at Fig. 2, it is easy to understand that the current increases in progressive segments, due to the presence of a new sub-station; therefore, the losses are different in each segment of the feeder. However, as the current flowing in each segment is the sum of the previous currents, recalling the approximation of unitary voltage amplitude at all the network buses, the generic active and reactive power losses $P_{j s_{i, i+1}}$ and 
$Q_{j s_{i, i+1}}$ in the interconnection between the $i^{\text {th }}$ and the $i^{\text {th }}+1$ PVS are given by:

$$
\left\{\begin{array}{l}
P_{j s_{i, i+1}}=R_{i, i+1}\left[\left(\sum_{k=1}^{i}\left(P_{i n v, k}-P_{j t_{k}}\right)\right)^{2}+\left(\sum_{k=1}^{i}\left(Q_{i n v, k}-Q_{j t_{k}}\right)\right)^{2}\right] \\
Q_{j s_{i, i+1}}=X_{i, i+1}\left[\left(\sum_{k=1}^{i}\left(P_{i n v, k}-P_{j t_{k}}\right)\right)^{2}+\left(\sum_{k=1}^{i}\left(Q_{i n v, k}-Q_{j t_{k}}\right)\right)^{2}\right]
\end{array}\right.
$$

being $R_{i, i+1}$ and $X_{i, i+1}$ the resistance and the reactance of the considered segment and $i=1 \ldots N_{i n v}$. As all voltages are considered equal to 1 p.u., it is possible to consider all the lines and cables shunt capacitances in parallel and connected to a 1 p.u. voltage, providing an "open-loop" total amount of reactive power per feeder given by:

$$
Q_{\text {tot }, C}=\sum_{k=1}^{N_{\text {iv }}} V_{k}^{2} \omega C_{k} \simeq \omega \sum_{k=1}^{N_{\text {inv }}} C_{k}
$$

Summing all the losses and recalling (4), it is possible to calculate the net active and reactive power produced by the generic feeder:

$$
\left\{\begin{array}{l}
P_{\text {feeder }}=\sum_{k=1}^{N_{i n v}} P_{i n v, k}-\sum_{k=1}^{N_{i n v}} P_{j t_{k}}-\sum_{i=1}^{N_{i n v}} P_{j s_{i, i+1}} \\
Q_{\text {feeder }}=\sum_{k=1}^{N_{i n v}} Q_{i n v, k}-\sum_{k=1}^{N_{i n v}} Q_{j t_{k}}-\sum_{i=1}^{N_{i n v}} Q_{j s_{i, i+1}}+\sum_{i=1}^{N_{i n v}} \omega C_{i}
\end{array}\right.
$$

Equations (6) can be identically written for all the feeders in parallel belonging to the same sub-field. In the case in which feeders are characterized by different layouts it is necessary to iterate equations (4)-(6) and then summing active and reactive powers obtained from each feeder. Active and reactive power at sub-field bus can be written as:

$$
\left\{\begin{array}{l}
P_{s f}=\sum_{r=1}^{N_{f}} P_{\text {feeder }, r} \\
Q_{s f}=\sum_{r=1}^{N_{f}} Q_{\text {feeder }, r}
\end{array}\right.
$$

It is now possible to assess the losses in the generic subfield interconnection and to determine the active and reactive power available from the considered sub-field ( $P_{M V s f}$ and

$$
\left.Q_{M V S f}\right) \text { : }
$$

$$
\left\{\begin{array}{l}
P_{M V s f}=P_{s f}-R_{M V S I}\left(P_{s f}^{2}+Q_{s f}^{2}\right) \\
Q_{M V S f}=Q_{s f}-X_{M V S I}\left(P_{s f}^{2}+Q_{s f}^{2}\right)+\omega C_{M V S I}
\end{array}\right.
$$

where $R_{M V S I}, X_{M V S I}$ and $C_{M V S I}$ are the resistance, reactance and the capacitance of the considered subfield interconnection (MVSI). The outputs of the feeder and subfield sub-system, namely the active and reactive power at the MV sub-field bus collector ( $P_{M V}$ and $\left.Q_{M V}\right)$, can be finally calculated as:

$$
\left\{\begin{array}{l}
P_{M V}=\sum_{k=1}^{N_{s f}} P_{M V s f, k} \\
Q_{M V}=\sum_{k=1}^{N_{s f}} Q_{M V s f, k}
\end{array}\right.
$$

Finally it is necessary to consider the common interconnection of the subfields with POI. In general, this structure accounts for a MV line or cable interconnection (MVCI) to the main MV/HV step-up transformer, usually provided with an Under Load Tap Changer (ULTC) device. Between the HV side of the transformer and the POI it is also possible to find a HV line, rather than a cable, (HVCI). In order to appreciate voltage variations caused by the PV plant active and reactive power the Thevenin equivalent of the System Operator network at the POI will be considered on the basis of its short circuit power. On this assumption, it is possible to calculate the active and reactive power and the voltage amplitude at the POI staring from the active and reactive power available at the MV collector bus (imposing the voltage magnitude behind the SO network short circuit impedance). The main step-up transformer is described with a pi representation, as depicted in Fig. 3, [7] and its shunt admittances are non-zero only when the transformer operates at a transformation ratio different from the rated one. The quantities appearing in Fig. 3 can be defined as [7]:

$$
\begin{gathered}
\dot{Y}_{40 t}=\frac{1}{\dot{Z}_{c c}}\left(\frac{1}{a_{n}^{2}}-\frac{1}{a_{n}}\right) \\
\dot{Y}_{30 t}=\frac{1}{\dot{Z}_{c c}}\left(1-\frac{1}{a_{n}}\right) \\
\dot{Z}_{t}=\frac{\dot{Z}_{c c}}{a_{n}}
\end{gathered}
$$

being $\dot{Z}_{c c}$ the short circuit impedance of the transformer and $a_{n}$ the ratio between the rated transformation ratio of the machine $K_{n}$ and the actual one $K$ :

$$
a_{n}=\frac{K_{n}}{K}
$$

In our modeling, aimed at verifying the steady state grid codes requirements, the quantity $a_{n}$ will be considered an independent input (as it is provided by a dedicated controller). Under this hypothesis, it is possible to draw the equivalent electric circuit of the common section of the plant as depicted in Fig. 3. Thanks to its simple and recursive structure, the network of Fig. 3 can be analytically solved expressing the $V_{5}$ as a function of the MV active power injection $P_{M V}$, MV reactive power injection $Q_{M V}$ and the TSO voltage $V_{1}$ as shown in the following calculations. Let us define:

$$
\begin{gathered}
\dot{Y}_{5}=\dot{Y}_{50 l} \\
\dot{Y}_{4}=\dot{Y}_{40 l}+\dot{Y}_{40 t} \\
\dot{Y}_{3}=\dot{Y}_{30 l}+\dot{Y}_{30 t} \\
\dot{Y}_{2}=\dot{Y}_{20 l} \\
\dot{Z}_{5}=\dot{Z}_{M V l i n e} \\
\dot{Z}_{4}=\dot{Z}_{t} \\
\dot{Z}_{3}=\dot{Z}_{H V l i n e} \\
\dot{Z}_{2}=\dot{Z}_{c c}
\end{gathered}
$$


and let us assume the voltage at the MV collector as the phase reference, i.e.:

$$
\dot{V}_{M V}=\dot{V}_{5}=V_{5} e^{j 0}
$$

with simple electrotechnical manipulations, one has that the voltage at bus $h(h=2 \ldots 5)$ and the current flowing in the longitudinal branches can be obtained as follows:

$$
\left\{\begin{array}{l}
\dot{V}_{h}=a_{v h} V_{5}+b_{v h} \frac{P_{M V}-j Q_{M V}}{V_{5}} \\
\dot{I}_{h}=a_{I h} V_{5}+b_{I h} \frac{P_{M V}-j Q_{M V}}{V_{5}}
\end{array}\right.
$$

where the coefficients of (23) can be obtained with the following recursive rule:

$$
\left\{\begin{array}{c}
a_{v, h-1}=a_{v, h}-\dot{Z}_{h} a_{I, h} \\
b_{v, h-1}=b_{v, h}-\dot{Z}_{h} b_{I, h} \\
a_{I, h-1}=a_{I, h}-\dot{Y}_{h-1} a_{v, h-1} \\
b_{I, h-1}=b_{I, h}-\dot{Y}_{h-1} b_{v, h-1} \\
a_{v, 5}=1 \\
b_{v, 5}=0 \\
a_{I, 5}=-\dot{Y}_{5} \\
b_{I, 5}=1
\end{array}\right.
$$

Now, writing the first of (24) for $h=1$, one obtains:

$$
\dot{V}_{1}=a_{v 1} V_{5}+b_{v 1} \frac{P_{M V}-j Q_{M V}}{V_{5}}
$$

Imposing that $\left|\dot{V}_{1}\right|=V_{T S O}$ one obtains a fourth order algebraic equation, whose solution provides the amplitude of the MV voltage $\bar{V}_{5}$. Substituting the value of $\bar{V}_{5}$ into (23) considering $h=2$ it is possible to calculate the voltage and current at the POI, $\dot{V}_{P O I}$ and $\dot{I}_{P O I}$. Active and reactive power injected at the POI are finally given by:

$$
\left\{\begin{array}{l}
P_{P O I}=\operatorname{Re}\left(\dot{V}_{P O I} \cdot \dot{I}_{P O I}^{*}\right) \\
Q_{P O I}=\operatorname{Im}\left(\dot{V}_{P O I} \cdot \dot{I}_{P O I}^{*}\right)
\end{array}\right.
$$

\section{VALIDATION}

The aim of this section is that of validating the bottom-up procedure for the evaluation of active power, reactive power and voltage at the POI starting from a given set of active and reactive power injections at the inverter sides against exact load flow calculations. The test bed plant is characterized by an installed power of $10 \mathrm{MWp}$ connected to a $115 \mathrm{kV}$ transmission network $(60 \mathrm{~Hz})$. The plant is designed to be composed by 6 identical PV Stations $\left(N_{i n v}=6\right)$ of rated apparent power of 1 MVA. PVSs are installed in one subfield ( $\left.N_{s f}=1\right)$ provided by 2 feeders $\left(N_{f}=2\right)$. Distance between progressive PVSs is considered to be constant.

Parameters used for the test case network are reported in Tab. I. Load flow calculations were performed imposing all the complex power injections at the LV sections of the PVSs and assuming the TSO bus bar as voltage reference $(1$ p.u. on a $115 \mathrm{kV}$ basis). In order to explore a wide area of operational assets, validation tests were performed in the following configurations of the PVSs:

1. Null production of active and reactive power $\left(P_{i n v}=0 \mathrm{MW}, Q_{i n v}=0 \mathrm{MVAr}\right.$ );

2. Active power production at unitary power factor $\left(P_{i n v}=0.8 \mathrm{MW}, Q_{i n v}=0 \mathrm{MVAr}\right)$;

3. Positive active and reactive power production ( $P_{i n v}=0.8 \mathrm{MW}, Q_{i n v}=0.3 \mathrm{MVAr}$ );

4. Positive active and negative reactive power production $\left(P_{i n v}=0.8 \mathrm{MW}, Q_{i n v}=-0.3 \mathrm{MVAr}\right.$ );

5. Null active power and positive reactive power $\left(P_{i n v}=0 \mathrm{MW}, Q_{i n v}=0.3 \mathrm{MVAr}\right.$ );

6. Null active power and negative reactive power $\left(P_{i n v}=0 \mathrm{MW}, Q_{i n v}=-0.3 \mathrm{MVAr}\right)$;

The validation results, reported in Tab. II, highlight a very good agreement with the exact values of the estimated quantities. As long as the POI active power is concerned, the maximum relative error is equal to almost $3 \%$, while the POI reactive power estimation error is equal to almost $1 \%$.

From these results it is possible to verify the increased accuracy with respect to those obtained by other equivalent calculation for renewable energy sauces characterized by an error which varies between $10 \%$ and $20 \%$ [8]-[9].

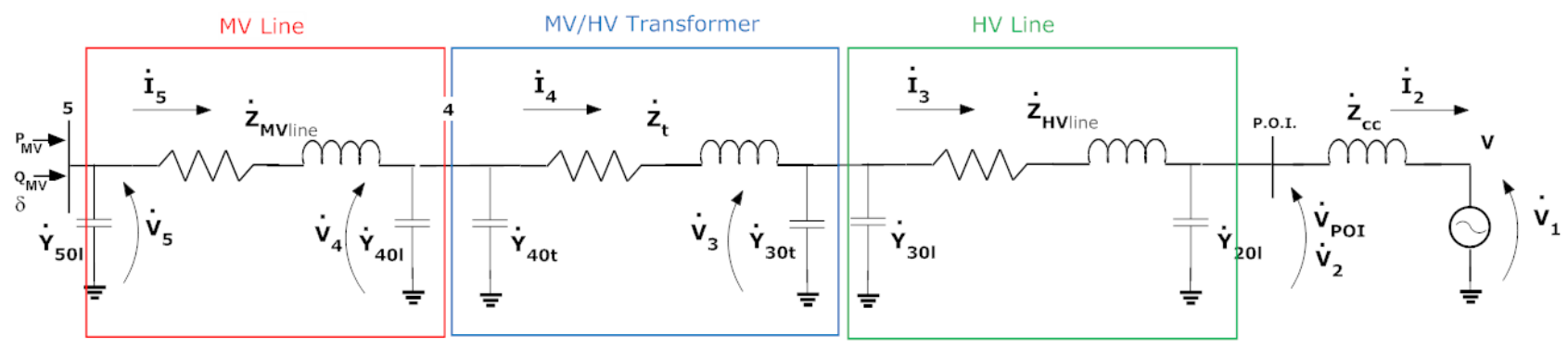

Fig. 3. Radial structure equivalent circuit 
Tab. I. Data of the test case power plant.

\begin{tabular}{|c|c|}
\hline \multicolumn{2}{|l|}{ PV Stations Data } \\
\hline Inverter rated AC Voltage & $0.48 \mathrm{kV}$ \\
\hline Inverter MPP active power & $0.855 \mathrm{MW}$ \\
\hline Inverter rating & $1 \mathrm{MVA}$ \\
\hline MV/LV transformer rated power & $1 \mathrm{MVA}$ \\
\hline Transformer ratio & $27.6 \mathrm{kV} / 0.48 \mathrm{kV}$ \\
\hline MV/LV transformer short circuit voltage & $4.42 \%$ \\
\hline \multicolumn{2}{|c|}{ Feeder Conductors Data } \\
\hline Medium distance among transformation cabins & $0.203 \mathrm{~km}$ \\
\hline Unitary resistance & $0.4073 \Omega / \mathrm{km}$ \\
\hline Unitary reactance & $0.12548 \Omega / \mathrm{km}$ \\
\hline Unitary capacity & $0.1689 \mu \mathrm{F} / \mathrm{km}$ \\
\hline \multicolumn{2}{|c|}{ Sub-field Interconnection Data } \\
\hline Connection length & $2.205 \mathrm{~km}$ \\
\hline Unitary resistance & $0.2076 \Omega / \mathrm{km}$ \\
\hline Unitary reactance & $0.4184 \Omega / \mathrm{km}$ \\
\hline Unitary capacity & $0.01045 \mu \mathrm{F} / \mathrm{km}$ \\
\hline \multicolumn{2}{|c|}{ MV Common Interconnection Data } \\
\hline Connection length & $0.3048 \mathrm{~km}$ \\
\hline Unitary resistance & $0.4073 \Omega / \mathrm{km}$ \\
\hline Unitary reactance & $0.1548 \Omega / \mathrm{km}$ \\
\hline Unitary capacity & $0.1689 \mu \mathrm{F} / \mathrm{km}$ \\
\hline Number of conductors in parallel & 5 \\
\hline \multicolumn{2}{|c|}{ Main Step-up Transformer Data } \\
\hline HV/MV transformer rated power & $20 \mathrm{MVA}$ \\
\hline Transformer ratio & $115 \mathrm{kV} / 27.6 \mathrm{kV}$ \\
\hline MV/LV transformer short circuit voltage & $7.50 \%$ \\
\hline \multicolumn{2}{|c|}{ HV Common Interconnection Data } \\
\hline Connection length & $0,208 \mathrm{~km}$ \\
\hline Unitary resistance & $0,478 \Omega / \mathrm{km}$ \\
\hline Unitary reactance & $0,57 \Omega / \mathrm{km}$ \\
\hline Unitary capacity & $0,0018 \mu \mathrm{F} / \mathrm{km}$ \\
\hline Short circuit power & $2.000 \mathrm{MVA}$ \\
\hline
\end{tabular}

It is also worth noticing that the various methodologies proposed in [8]-[9] do not allow having any information about the voltage at the POI. Tab. III reports the results related to the POI voltage calculation once again characterized by small relative errors.

Tab. II - Validation results for active and reactive power at the POI

\begin{tabular}{|c|c|c|c|c|c|c|}
\hline Case & $\begin{array}{c}\boldsymbol{P}_{\text {POI }} \\
{[\mathbf{k W}]}\end{array}$ & $\begin{array}{c}\boldsymbol{Q}_{P O I} \\
{[\mathbf{k V A r}]}\end{array}$ & $\begin{array}{c}\boldsymbol{P}_{P O I_{-} L F} \\
{[\mathbf{k W}]}\end{array}$ & $\begin{array}{c}\boldsymbol{Q}_{P O I_{-} L F} \\
{[\mathbf{k V A r}]}\end{array}$ & $\begin{array}{c}\boldsymbol{\varepsilon} P_{P O I} \\
{[\%]}\end{array}$ & $\begin{array}{c}\boldsymbol{\varepsilon} Q_{P O I} \\
{[\%]}\end{array}$ \\
\hline 1 & $-0,01$ & 200,52 & $-0,01$ & 200,73 & $0,00 \%$ & $0,10 \%$ \\
\hline 2 & $9.528,42$ & $-598,16$ & $9.529,03$ & $-597,23$ & $0,01 \%$ & $0,16 \%$ \\
\hline 3 & $9.519,75$ & $2.912,42$ & $9.523,09$ & $2.943,53$ & $0,04 \%$ & $1,06 \%$ \\
\hline 4 & $9.516,95$ & $-4335,53$ & $9.514,19$ & $-4.372,08$ & $0,03 \%$ & $0,84 \%$ \\
\hline 5 & $-10,41$ & $3.686,88$ & $-10,16$ & $3.694,00$ & $2,46 \%$ & $0,19 \%$ \\
\hline 6 & $-9,77$ & $-3.511,34$ & $-10,08$ & $-3.520,38$ & $3,08 \%$ & $0,26 \%$ \\
\hline
\end{tabular}

Tab. III - Validation results for voltage amplitude at the POI

\begin{tabular}{|c|c|c|c|}
\hline Case & $\begin{array}{c}\mathbf{V}_{\text {POI }} \\
{[\mathbf{k V}]}\end{array}$ & $\begin{array}{c}\mathbf{V}_{\text {POL_LF }} \\
{[\mathbf{k V}]}\end{array}$ & $\begin{array}{c}\boldsymbol{\varepsilon V _ { \text { POI } }} \\
{[\%]}\end{array}$ \\
\hline 1 & 115.012 & 115.012 & $0.000 \%$ \\
\hline 2 & 114.964 & 114.965 & $0.001 \%$ \\
\hline 3 & 115.166 & 115.168 & $0.002 \%$ \\
\hline 4 & 114.749 & 114.747 & $0.002 \%$ \\
\hline 5 & 115.212 & 115.212 & $0.000 \%$ \\
\hline 6 & 114.798 & 114.797 & $0.001 \%$ \\
\hline
\end{tabular}

\section{CONSLUSIONS}

This work proposed a novel procedure for the evaluation of active power, reactive power and voltage at the Point of Interconnection of a large photovoltaic power plant based on simple and available information regarding the plant main components and layout. The usefulness of such a procedure lays in the improving requirements of TSOs and DSOs for the interconnection of new power plants to their electricity grids which introduced new aspects to deal with in the design of PV plants. In this context the availability of a simple but effective tool which allows verifying the compliancy of a proposed power plant layout with well-defined grid code requirements represents a useful support for the bidding and pre-design phase of the power plant realization. This approach allows verifying the compliancy of the power plant capabilities with a specific grid code requirement and finding the solution that best fits its request from a technical an economical point of view. The procedure may also be used in case of mismatch of the power plant capability to adjust the pre-design layout without using dedicated load flow software. The accuracy of the proposed methodology has been tested on a realistic structure of photovoltaic plant and compared with the results of a complete load flow. This comparison highlighted an optimal agreement of the estimation error which is limited between $1 \%$ and $3 \%$. Future developments will concern the improvement of the proposed methodology with the calculation of some internal electric parameters of the plant (voltages and currents) to give some preliminary indications about the actual feasibility of the proposed layout. The procedure may also be used for the verification of specific TSO/DSO regulation requiems from a steady state point of view.

\section{REFERENCES}

[1] J.A., Momoh, Yan Xia; G.D., Boswell "An Approach to Determine Distributed Generation Benefits in Power Networks" 40th North American Power Symposium, 2008, DOI 10.1109/NAPS.2008.5307395.

[2] Davda, A.T., Parekh, B.R. "System impact analysis of Renewable Distributed Generation on an existing Radial Distribution Network" IEEE Electrical Power and Energy Conference 2012, 10-12 Oct. 2012 Halifax, NS, Canada, pp. 128-132, DOI 10.1109/EPEC.2012.6474936.

[3] CEI 0-16 Reference technical rules for the connection of active and passive consumers to HV and MV electrical networks, Issued on 2012.

[4] IESO Power to Ontario on Demand Market Rules - Chapter 4 Grid Connection Requirements Issue Data: June 5, 2013.

[5] A. Bonfiglio, M. Brignone, F. Delfino, M. Invernizzi, F. Pampararo, R. Procopio, "A Centralized Control Algorithm for Grid-Connected PV Units aimed at Voltage Support and Energy Efficiency Improvement" UPEC 2012, 4-7 September, London, UK, DOI 10.1109/UPEC.2012.6398657.

[6] A. Bonfiglio, M. Brignone, F. Delfino, R. Procopio "Optimal Control and Operation of Grid-Connected Photovoltaic Production Units for Voltage Support in Medium Voltage Networks" IEEE Transaction on Sustainable Energy DOI 10.1109/TSTE.2013.2280811.

[7] Milano, F. Power System Modelling and Scripting. Springer London Dordrecht Heidelberg New York, 2010, ISSN 1612-1287.

[8] Muljadi, E., Butterfield, C.P., Ellis, A., Mechenbier, J.; Hochheimer, J., Young, R.; Miller, N.; Delmerico, R ; Zavadil, R ; Smith, J.C. "Equivalencing the collector system of a large wind power plant" IEEE Power Energy Society General Meeting, 2006, June 18-22, Montreal, Quebec, Canada, DOI 10.1109/PES.2006.1708945.

[9] A. Bonfiglio, F. Delfino, M. Invernizzi, P. Serra, R. Procopio "Criteria for the Equivalent Modeling of Large Photovoltaic Power" IEEE Power and Energy Society General Meeting 2014, July 27-31, Washington DC, USA, DOI 10.1109/PESGM.2014.6939183. 